\title{
Arteria Subclavia Aberrante
}

\author{
Aberrant Subclavian Artery
}

*Oscar Inzunza \& **Álvaro Burdiles

INZUNZA, O. \& BURDILES, A. Arteria subclavia aberrante. Int. J. Morphol., 28(4):1215-1219, 2010.

RESUMEN: Se estudiaron las variaciones en el origen y trayecto de la arteria subclavia derecha aberrante, observada en un cadáver utilizado para docencia de anatomía humana. En este caso, el arco aórtico da origen a cuatro ramas separadas, que de derecha a izquierda son: arteria carótida común derecha, arteria carótida común izquierda, arteria subclavia izquierda y arteria subclavia derecha. Desde este origen, la arteria subclavia derecha transcurre por detrás de la tráquea y del esófago, a la altura del cuerpo vertebral de T III, para luego retomar su trayecto normal hacia la raíz del cuello. Se midieron los diámetros del arco aórtico y de sus ramas. Asimismo, se consignaron los díametros y la distancia de origen de ambas arterias vertebrales, desde las arterias subclavias. Esto se realizó con el objeto de analizar posibles asimetrías importantes relacionadas con la aparición de esta variación. En el texto se discuten las probables razones embriológicas que permiten explicar el origen anómalo de la arteria subclavia derecha.

PALABRAS CLAVE: Arteria subclavia derecha aberrante; Arteria lusoria; Variación anatómica.

\section{INTRODUCCIÓN}

El estudio de las variaciones anatómicas es relevante para quienes practicamos las ciencias morfológicas y para quienes trabajan en el diagnóstico de imágenes y en cirugía. Si bien muchas de estas variaciones pueden pasar desapercibidas durante la vida de un sujeto, en la mayoría de ellas existen implicancias de interés clínico que vale la pena estudiar (Inzunza et al., 1996a, 1996b, 2006; Inzunza \& Burdiles, 2010).

La anatomía normal del arco aórtico se caracteriza por el nacimiento de 3 ramas cuyo orden de irrupción es: tronco braquiocefálico, arteria carótida común izquierda y arteria subclavia izquierda. En condiciones normales el tronco braquiocefálico se dirige hacia arriba y afuera, dispuesto por dorsal de la vena braquiocefálica izquierda y por ventral de la tráquea. A la altura de la articulación esternoclavicular derecha, el tronco se divide en sus terminales, la arteria carótida común y la arteria subclavia (Testut \& Latarjet, 1960; Moore, 1980).

En el caso motivo de esta presentación se observa una variación anatómica del arco aórtico con cuatro ramas, detectándose el origen separado de la carótida común dere- cha y la subclavia derecha, teniendo esta última un trayecto aberrante retroesofágico y constituyendo la rama más distal del arco aórtico. Esta situación anómala es conocida como "arteria lusoria" (Fazan et al., 2003).

La observación accidental de esta variación fue realizada en un cadáver de sexo masculino, de 65 años de edad y con antecedentes de sepsis por Gram (+), bronconeumonía y alcoholismo crónico como causales de muerte, utilizado para fines docentes en el Departamento de Anatomía de la Escuela de Medicina de la Pontificia Universidad Católica de Chile. Este hallazgo resulta especialmente atractivo por las implicancias embriológicas que permiten su explicación.

\section{MATERIAL Y MÉTODO}

En un cadáver de sexo masculino, de 65 años de edad cuya causa de muerte fue sepsis por Gram (+), bronconeumonía y alcoholismo crónico, utilizado para fines docentes desde el año 1998, se realizó una disección de mediastino superior y se identificaron las ramas del arco aórtico, la tráquea y el esófago; siguiendo el trayecto de la arteria subclavia derecha aberrante hasta su continuidad con la arteria axilar. Luego, se procedió a inyectar silicona en

\footnotetext{
* Profesor Titular, Departamento de Anatomia. Escuela de Medicina, Pontifícia Universidad Católica de Chile.

** Profesor Asistente, Departamento de Radiología. Escuela de Medicina, Pontifícia Universidad Católica de Chile.
} 
las arterias de la región estudiada para medir con piedemetro el calibre de las ramas del arco aórtico a diferentes niveles, con el objetivo de emular los diámetros in vivo del sujeto. Se puso especial cuidado en llenar y medir ambas subclavias desde su origen hasta el nacimiento de la arteria vertebral, rama de su primera porción. Del mismo modo, se identificó en la región lateral derecha del cuello la situación del nervio laríngeo inferior y de la arteria tiroidea inferior.

\section{RESULTADOS}

La longitud de la arteria subclavia derecha aberrante desde su nacimiento en el arco aórtico hasta el punto donde da origen a la arteria vertebral es de $52,1 \mathrm{~mm}$. Su diámetro a nivel del arco aórtico es de 10,8 mm, dimensión que se reduce a 10,2 mm luego de pasar por detrás del esófago. Por otro lado, el diámetro de la arteria subclavia izquierda a nivel del arco aórtico es de $10,5 \mathrm{~mm}$ y, a $37 \mathrm{~mm}$ de este punto da origen a la arteria vertebral.

La medición del calibre de las arterias vertebrales, en el punto de origen en las arterias subclavias arrojó valores del orden de $3,5 \mathrm{~mm}$ en el lado derecho y $6,5 \mathrm{~mm}$ en el lado izquierdo.

La arteria carótida común derecha, nacida directa-

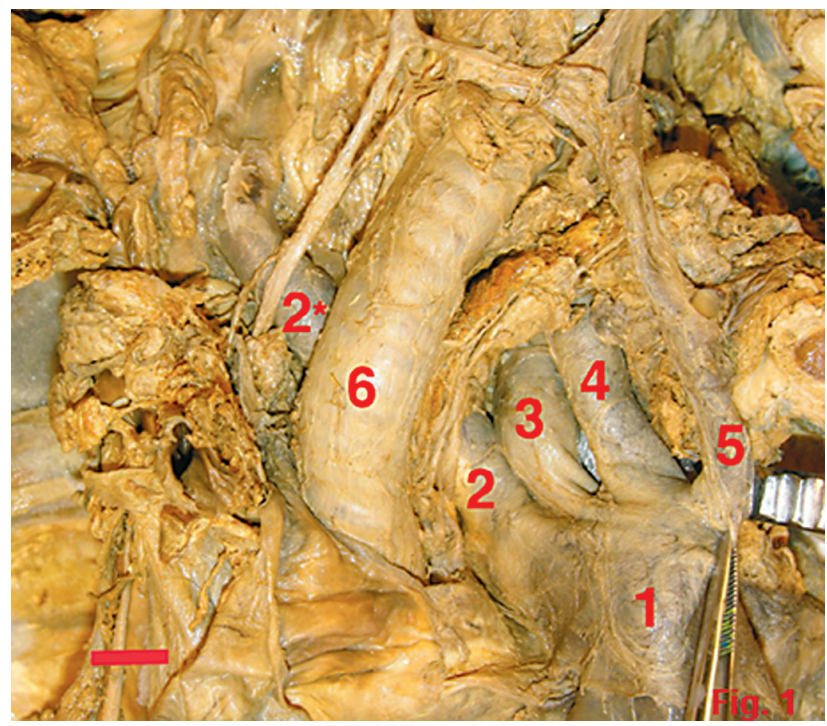

Fig. 1. Fotografía del arco aórtico (1) fuertemente traccionado hacia la antímera izquierda que presenta el origen cuatro ramas, que de izquierda a derecha corresponden a: arteria subclavia derecha (2), arteria subclavia izquierda (3), carótida común izquierda (4) y carótida común derecha (5). La subclavia aberrante pasa por detrás de la traquea (6) y de esófago siguiendo su trayecto $\left(2^{*}\right)$ hacia la raíz del cuello. Barra $=1 \mathrm{~cm}$. mente desde la aorta, presentó un diámetro inicial de 8,7 $\mathrm{mm}$, con una extensión de 144,5 mm. hasta su bifurcación. Por su parte, el diámetro inicial de la arteria carótida común izquierda fue de $9,15 \mathrm{~mm}$, y su extensión total fue de 147 $\mathrm{mm}$.

El diámetro del arco aórtico en casi toda su longitud fue de $30,1 \mathrm{~mm}$. El diámetro del esófago a cefálico y a caudal a la arteria lusoria alcanzó valores de $22,45 \mathrm{~mm}$ y 16,4 $\mathrm{mm}$ respectivamente.

Por su parte, los diámetros de la tráquea a cefálico, a nivel y a caudal de la arteria lusoria fueron, respectivamente, de $18,45 \mathrm{~mm}, 20,3 \mathrm{~mm}$ y $19,3 \mathrm{~mm}$ (Fig. 1).

En la región cérvico lateral derecha se observaron variaciones en el origen y en el trayecto de la arteria tiroidea inferior y del nervio laríngeo recurrente. Así, la arteria

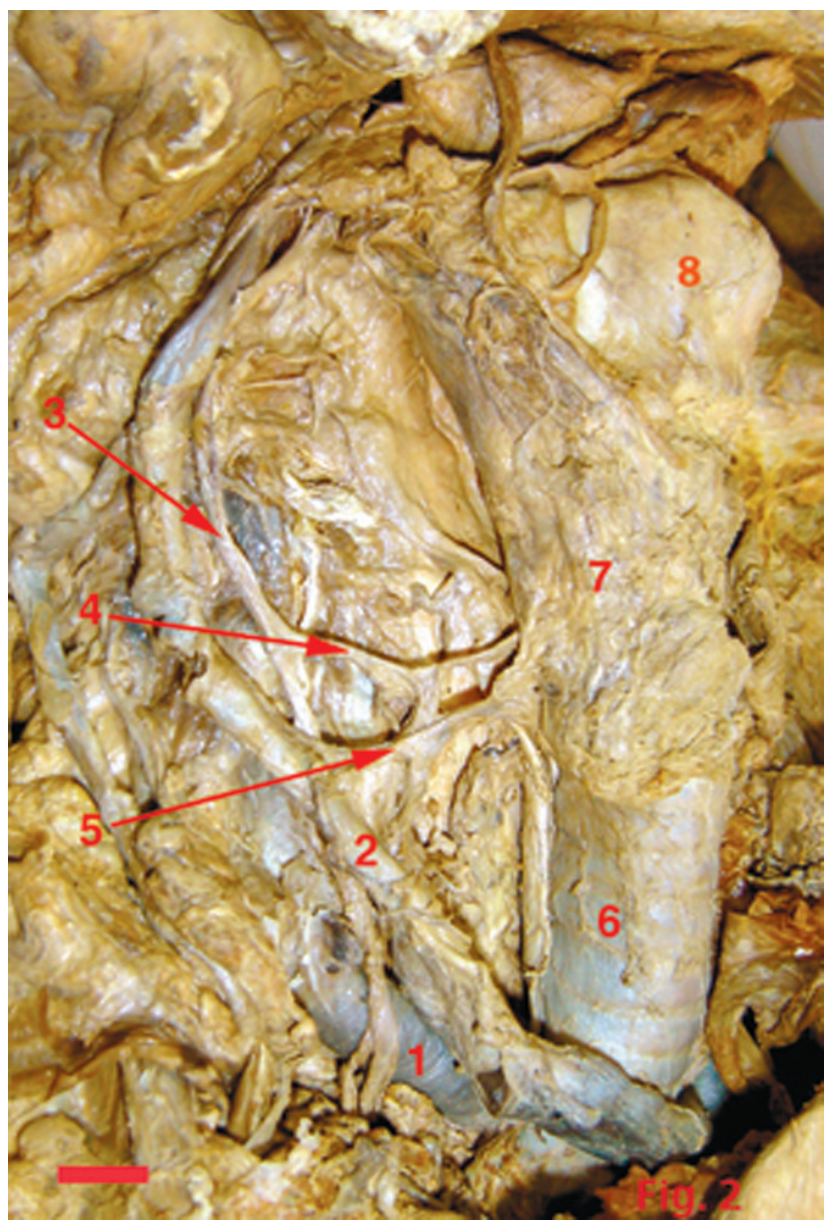

Fig. 2. Fotografía de la región cervical derecha del mismo cadáver de la Fig. 1, que muestra el origen y trayecto de el nervio laríngeo recurrente (4) y la arteria tiroidea inferior (5). 1= Arteria subclavia, $2=$ Arteria carótida común, $3=$ Nervio vago, $6=$ Traquea, $7=$ Glándula tiroides, $8=$ Cartilago tiroides. Barra $=1 \mathrm{~cm}$. 
tiroidea inferior derecha se origina desde la carótida común, a 55,8 mm. del origen de esta en el arco aórtico, siguiendo un trayecto oblicuo hacia arriba y medial. Del mismo modo, el nervio laríngeo recurrente derecho se origina desde el nervio vago a 40,1 $\mathrm{mm}$. del borde inferior de la subclavia aberrante, tomando una dirección perpendicular hacia medial convergiendo junto con la arteria tiroidea inferior a nivel del polo inferior de la glándula tiroides (Fig. 2).

\section{DISCUSIÓN}

Los múltiples cambios que sufre el patrón de simetría vascular de los arcos aórticos para desarrollar el modelo

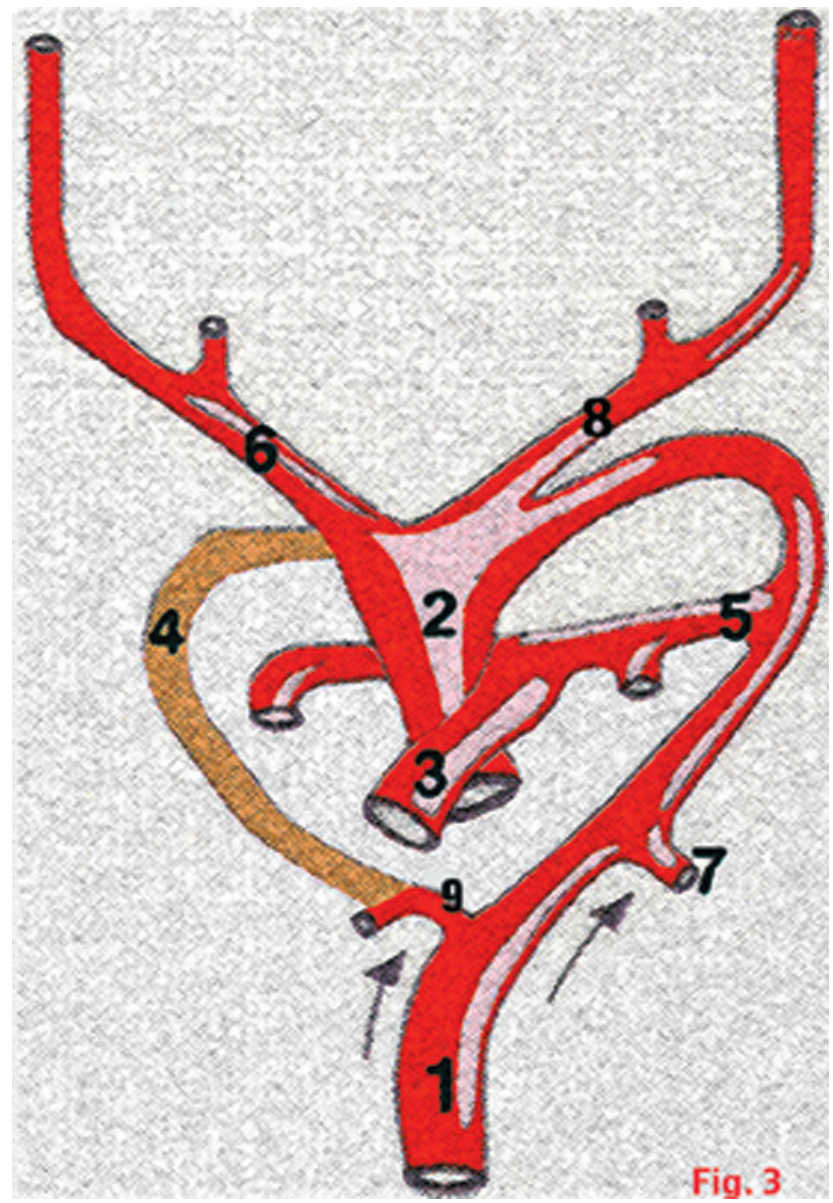

Fig. 3. Esquema modificado de Moore y Persaud que ilustra la probable explicación esta anomalía, donde el cuarto arco aórtico y la porción craneal de la aorta dorsal derecha (4) involucionan, de modo que la subclavia derecha se forma a partir de la séptima arteria intersegmentaria derecha (9); las flechas indican el sentido en que son arrastradas estas ramas, dejando a la subclavia derecha como la última rama del arco aórtico. $1=$ Aorta dorsal, $2=$ Aorta ascendente, $3=$ Tronco pulmonar, 5= Ducto arterioso, $6=$ Carótida común, $7=$ Séptima arteria intersegmentaria izquierda, $8=$ Arteria carótida común izquierda. arterial asimétrico del adulto, implican un complejo proceso de regresiones y remodelaciones. Alteraciones en estas transformaciones pueden provocar variaciones anatómicas complejas, las que si bien pueden pasar desapercibidas durante el período fetal también pueden llegar a ser incompatibles con la vida. En este contexto, las variaciones del arco aórtico ofrecen una gran variabilidad de presentación, siendo la mayoría de ellas detectadas incidentalmente. Así, en un temprano reporte del siglo pasado, publicado en el primer número de los Archivos Chilenos de Morfología por el Dr. Nicolás Muñoz Hidalgo (Muñoz, 1933), quien grafica elegantemente al menos siete tipos de variaciones del arco aórtico observadas en una serie de 300 disecciones de cadáveres femeninos.

La arteria subclavia derecha aberrante de trayecto retroesofágico representa una variedad de anomalía del arco aórtico que puede ser incluida en la categoría de "anillo vascular" (Carrizo \& Marjani, 2004), siendo detectada entre el 0,4 y el 2,0\% de las autopsias (Stone et al., 1990), constituyendo la malformación más común del arco aórtico (Richardson et al., 1980).

Si bien en la mayoría de los casos esta anomalía es asintomática, lo que explica su hallazgo incidental, el anillo vascular que encierra a la traquea y al esófago puede generar una compresión extrínseca de este último conducto, apareciendo el síntoma de disfagia (Fig. 4). Este fenómeno fue descrito por primera vez en 1794, por el médico londinense David Bayford, quien originalmente la describió como una "disfagia por un capricho de la naturaleza", siendo referida como disfagia lusoria, de "lusus naturae" (Bayford, 1794); o, también, como disfagia de Bayford-Autenrieth.

La literatura especializada reporta que la arteria subclavia derecha aberrante de trayecto retroesofágico es frecuentemente observada en mujeres y en niños con síndrome de Down (Momma et al., 1999; Fazan et al.; Carrizo \& Marjani). Además, algunos estudios han asociado a esta anomalía con el desarrollo de patologías trombo-isquémica de la extremidad superior derecha (Akers et al., 1991; Boas et al., 2002). Por otro lado, una rara pero importante complicación de esta anomalía es el desarrollo de una fístula arterio-esofágica, evento generalmente fatal, situación observada por Feugier et al. (2003) en 11 casos.

La presencia de estas variaciones anatómicas, observadas en cadáveres utilizados con fines docentes, son especialmente atractivas para los alumnos y a nosotros nos ha motivado darlas a conocer (Inzunza et al.) para resaltar lo formativo de la disección anatómica; método éste no superado para aprender, realmente, anatomía humana (Bravo \& Inzunza, 1995). 
Esta variación en particular, la arteria lusoria, es especialmente atractiva para el morfólogo, quien busca en la embriología la explicación del fenómeno observado. A este respecto, Edwards (1960) sugiere que el origen anómalo de la arteria subclavia derecha puede ser explicado por la involución del IV arco aórtico derecho, con la persistencia de la séptima arteria intersegmental derecha que mantiene su conexión con la aorta dorsal (Fig. 3). A medida que se forma el arco aórtico, este punto de unión es arrastrado cranealmente, adoptando una posición final como la cuarta rama del arco aórtico, lateral a la subclavia izquierda (Larsen, 1998; Moore \& Persaud, 1999).

Esta variación anatómica también afecta al nervio laríngeo recurrente derecho el cual, que en condiciones normales queda enganchado en el IV arco aórtico derecho, al no existir este vaso adopta un trayecto directo hacia la laringe (Moore;
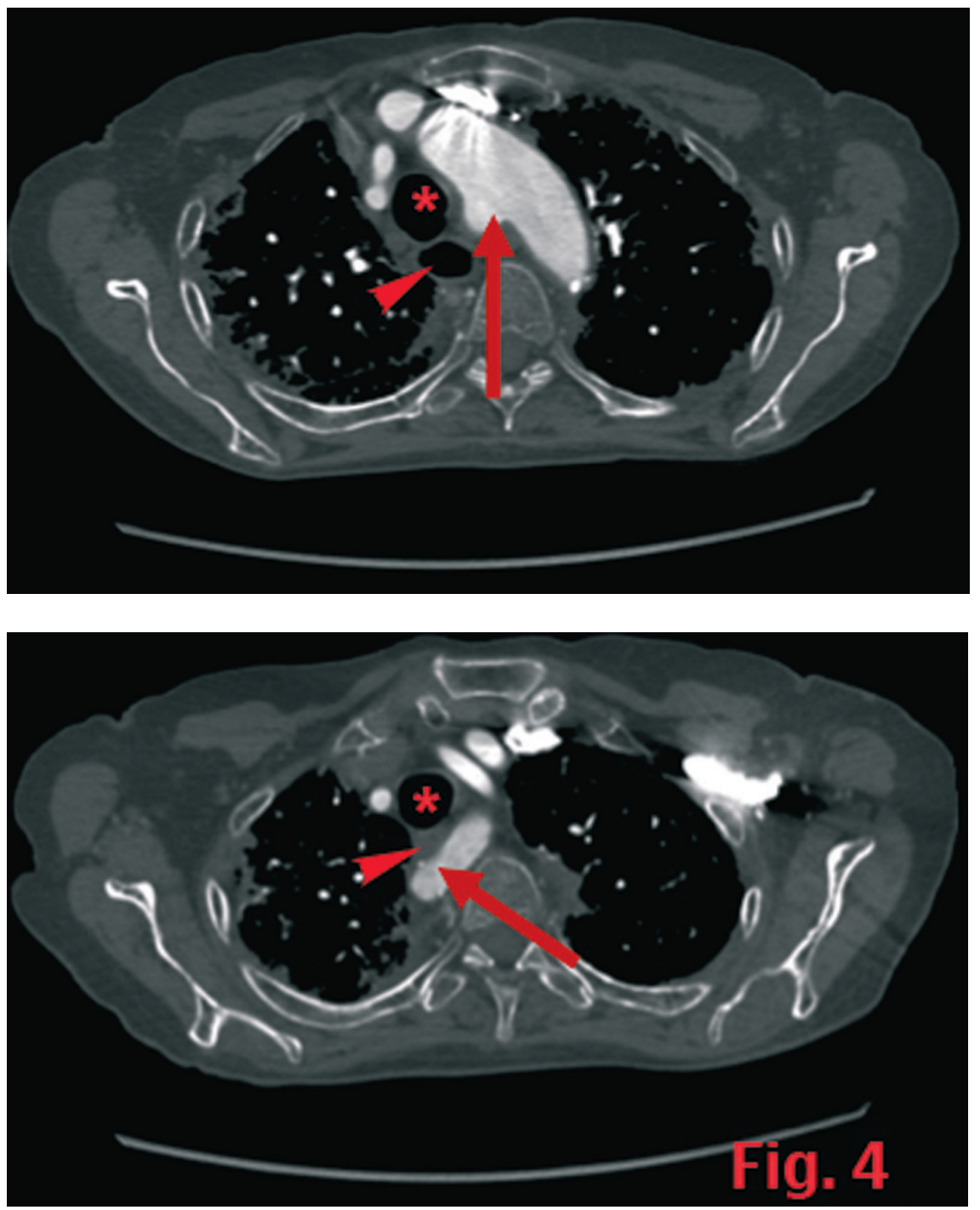

Fig. 4. Tomografía axial computada del mediastino superior. La imagen superior muestra el origen, en el arco aórtico, de la arteria subclavia derecha (flecha). A este nivel, la arteria se dispone a la izquierda de la traquea $\left(^{*}\right)$ y del esófago que aparece distendido con aire (punta de flecha). La imagen inferior muestra como la arteria subclavia derecha, de origen aberrante, se dispone por dorsal de la traquea y del esófago.
Moore \& Persaud). Del mismo modo, en el caso que aquí describimos, se observa una variación en el origen de la arteria tiroidea inferior derecha, elemento que se origina en la carótida común derecha, tomando una trayectoria convergente junto con el nervio laríngeo recurrente hacia el polo inferior de la glándula; variación vascular que no aparece descrita previamente en relación con la arteria lusoria.

INZUNZA, O. \& BURDILES, A. Aberrant subclavian artery. Int. $\boldsymbol{J}$. Morphol., 28(4):1215-1219, 2010.

SUMMARY: The anatomical variations involving the origin and passage of the right aberrant subclavian artery were studied, based on findings in a cadaver used for human anatomy teaching. In this case, the aortic arch gives rise to four separated branches, from right to left: right common carotid artery, left common carotid artery, left subclavian artery and right subclavian artery. From its origin, the right subclavian artery runs behind the trachea and esophagus, at the level of the third thoracic vertebrae, afterwards it reassumes its normal passage to the neck. The diameter of the aortic arch and its four branches were measured. In both vertebral arteries the diameter and distance from its origin in the subclavian arteries to the origin of the subclavian arteries were measured, with the purpose of analyzing potential important asymmetries. The probable embryologic explanations for this anomaly are discussed.

KEY WORDS: Right aberrant subclavian artery; Arteria lusori; Anatomical variation. 


\section{REFERENCIAS BIBLIOGRÁFICAS}

Akers, Jr. D.L.; Fowl, R.J.; Plettner, J.; Kempczinski, R.F. Complications of Anomalous Origin of the Right Subclavian Artery: Case Report and Review of the Literatura. Ann. Chir. Vasc., 5:358-88, 1991.

Bayford, D. An account of a singular case of obstructed deglutition. Memoirs Med. Soc. London, 2:275-86, 1794.

Boas, N.; Desmoucelle, F.; Bernadet, V. \& Rancesch, J. C. Rare cause of acute ischemia of the right upper extremity: thrombosis of a retroesophageal subclavian artery. Ann. Vasc. Surg., 16:387-90, 2002.

Bravo, H. \& Inzunza, O. Evaluación de algunos programas computacionales en la enseñanza de Anatomía y Neuroanatomía de la Facultad de Medicina de la Pontificia Universidad Católica de Chile. Rev. Chil. Anat., 13:79-86, 1995.

Carrizo, G. J. \& Marjani, M. A. Dysphagia lusoria caused by an aberrant right subclavian artery. Tex. Heart Inst. J., 31:168-71, 2004.

Edwards, J. E. Congenital malformations of the Heart and great vessels. Section H. Malformations of the thoracic aorta. In: Gould, S. E. (Ed.) Pathology of the Heart, $2^{\text {nd }}$ ed. Springfield, Charles C. Thomas, 1960. pp.391462.

Fazan, V.; Ribeiro, R.; Ribeiro, J. \& Rodrigues, O. Right Retroesophageal Subcllavian Artery. Acta Cir. Bras., 18:54-6, 2003.

Feugier, P.; Lemoine, L.; Gruner, L.; Bertin-Maghit, M.; Rousselet, B. \& Chevalier, J. M. Artrioesophageal fistula: A rare complication of Retroesophageal Subclavian Arteries. Ann. Vasc. Surg., 17:302-5, 2003.

Inzunza, O.; Prado, M. \& Valenzuela, A. Variación del origen de la arteria coronaria derecha. Rev. Chil. Cs. Med. Biol., 6:11-4, 1996a.

Inzunza, O.; Valenzuela, A. \& Prado, M. Anastomosis anómala entre las arterias hepática y mesentérica superior. Rev. Chi.l Cs. Biol., 6:65-8, 1996 b.

Inzunza, O. \& Burdiles, A. Arteria vertebral izquierda aberrante. Int. J. Morphol., 28(1):209-12, 2010.
Inzunza, O.; Ortega, W.; Berrocal, C. \& Vargas, A. Variación anatómica de la valva atrioventricular derecha. Int. J. Morphol., 24:49-52, 2006.

Larsen, W. J. Essentials of human embryology. New York, Churchill Livingstone, 1998. pp.146-7.

Momma, K.; Matsuoka, R. \& Takao, A. Aortic Arch Anomalies Associated with Chromossome 22q11 Deletion (CATCH 22). Pediatr. Cardiol., 20:97-102, 1999.

Moore, K. L. Clinically oriented anatomy. Baltimore, Willians \& Wilkins, 1980. pp.95-6.

Moore, K. L. \& Persaud, T. V. Embriología Clínica. México D.F., Mc Graw-Hill Interamericana, 1999. pp.406-13.

Muñoz, N. Variedades de cayado aórtico en la mujer. Arch. Chil. Morfol., 1:27-37, 1933.

Richardson, J. V.; Doty, D. B.; Rossi, N. P. \& Ehrenhaft, J. L. Operation for Aortic Arch Anomalies. Ann. Thorac. Surg., 31:426-32, 1980.

Stone, W. M.; Brewster, D. C. \& Moncure, A. C. Aberrant Right subclavian artery: varied presentations and management options. J. Vasc. Surg., 11:812-7, 1990.

Testut, L. \& Latarjet, A. Anatomía humana. Barcelona, Salvat, 1960. pp.202-6.

Dirección para correspondencia:

Prof. Dr. Oscar Inzunza

Departamento de Anatomía Normal

Escuela de Medicina,

Pontificia Universidad Católica de Chile

CHILE

Teléfono 56-2-354 3076

Email: oinzunza@med.puc.cl

Recibido : 12-07-2010

Aceptado: 22-09-2010 
